\title{
Azobenzene sulphonic dye photoalignment as a means to fabricate liquid crystalline conjugated polymer chain-orientation-based optical structures
}

\author{
Haoran Zhang, ${ }^{\#}$ Lingling Ma, Qian Zhang, Yuping Shi, Yueting Fang, Ruidong Xia, * Wei \\ Hu, * Donal D. C. Bradley*
}

${ }^{\#}$ H. Zhang, L. Ma and Q. Zhang contributed equally to this work.

H. Zhang, Q. Zhang, Y. Fang, Prof. R. Xia

Key Laboratory for Organic Electronics \& Information Displays (KLOEID), JiangsuSingapore Joint Research Center for Organic/Bio Electronics \& Information Displays, Institute of Advanced Materials (IAM), Nanjing University of Posts and Telecommunications, 9 Wenyuan Road, Nanjing 210046, China

E-mail: iamrdxia@,njupt.edu.cn

L. Ma, Prof. W. Hu

National Laboratory of Solid-State Microstructures, Collaborative Innovation Center of Advanced Microstructures, and College of Engineering and Applied Sciences, Nanjing University, Nanjing 210093, China

E-mail: huwei@,nju.edu.cn

Y. Shi

Department of Engineering Science, University of Oxford, Parks Road, Oxford OX1 3PJ, UK

Prof. R. Xia

The International School of Advanced Materials, School of Material Science and Engineering, South China University of Technology, 381 Wushan Road, Tianhe District, Guangzhou, 510640, China

E-mail: rxia@scut.edu.cn

Prof. D. D. C. Bradley

Physical Science and Engineering Division, King Abdullah University of Science and Technology, Thuwal 23955-6900, KSA

and Department of Physics, University of Oxford, Parks Road, Oxford OX1 3PU, UK

E-mail: Donal.Bradley@kaust.edu.sa

Keywords: Liquid crystalline conjugated polymer, F8BT and Red-F, SD1 photoalignment, Chain orientation optical structures, Refractive index modulation

Abstract: The use of a non-contact photoalignment method to fabricate in-plane optical structures, defined by the local uniaxial ordering of liquid crystalline conjugated polymer chains, is reported. Molecular orientation is demonstrated for both green-light-emitting fluorene-benzothiadiazole alternating copolymer F8BT and F8BT/red light emitting complex copolymer Red-F binary blend films deposited on a well-known azobenzene sulphonic dye photoalignment material SD1. Absorption anisotropy ratios of up to 9.7 are readily achieved 
for $150 \mathrm{~nm}$ thickness F8BT films. Spatial pattern definition, afforded by masking the UV polarized light exposure of the photoalignment layer, allowed the fabrication of optical structures with a resolution down to the micron scale. The alignment process has been further extended to enable the serial, independent orientation of films deposited on top of each other and to permit the molecular orientation to follow curvilinear patterns. In the former case this allows F8BT bilayer structures to be fabricated that show even higher absorption anisotropy ratios, up to $\sim 12$, close to the theoretical limit for the previously deduced $\sim 22^{\circ}$ optical transition dipole moment angle relative to the chain axis.

\section{Introduction}

Mainchain liquid crystalline conjugated polymers (LCCPs) ${ }^{[1-9]}$ offer the opportunity to reveal and utilize the natural optical and electrical anisotropy of long chain soluble semiconductors through molecular level orientation on suitable alignment layers. ${ }^{[9-18]}$ This has allowed the demonstration of polarized polymer light emitting diodes (LEDs), ${ }^{[7,12,19]}$ highly polarized microcavity emission sources, ${ }^{[20]}$ enhanced thin film optical gain media and microcavity lasers, ${ }^{[21-23]}$ and optimized charge carrier transport, including within field effect transistor (FET) device structures. ${ }^{[24-26]}$

Rubbed alignment layers have, however, several potential drawbacks in relation to the resulting surface roughness, the presence of debris and electrostatic charges, and a limited ability to spatially pattern the subsequent liquid crystal orientation. Transfer printing can allow directional/local placement of oriented films but this approach is not conducive to straightforward fabrication. ${ }^{[27]}$ Non-contact photoalignment provides a different approach and a variety of polymeric and cross-linkable alignment layer materials have been used to align LCCPs and related oligomers for LED and FET applications. ${ }^{[28-32]}$ For example, polarized electroluminescence was demonstrated by Sainova et $a l,{ }^{[29]}$ albeit with relatively modest 
efficiency $(\leq 0.1 \mathrm{~cd} / \mathrm{A})$ and at relatively high drive voltage $(\geq 10 \mathrm{~V})$, additionally requiring doping of the alignment layer with a significant fraction of a hole transport molecule. The reported photoalignment approach did, encouragingly, allow selective definition of spatial patterns through a multistep, optical rewriting process; a 300 mesh transmission electron microscope grid was used as mask. ${ }^{[29]}$ Further improvements are needed in order that photoalignment can become a routine patterning method for LCCP chain-orientation-based optical structures. In particular, a more readily processed alignment layer material with suitable optical and electrical characteristics is desirable.

In this paper we report the first use for LCCP chain-orientation of a well-studied sulfonic azo dye SD1 (see Figure 1(a) for chemical structure), previously used to good effect as a photoalignment layer for liquid crystal displays and other optical structures. ${ }^{[33-37]}$ We focus on the green-light emission poly(9,9-dioctylfluorene-co-benzothiadiazole) (F8BT) LCCP (Figure 1 (b)) and a 90wt $\%$ host: $10 \mathrm{wt} \%$ guest binary blend with the red-light emission complex copolymer Red F (comprising 9,9-dioctylfluorene (50\%), benzothiadiazole (40\%), triarylamine (5\%) and thiophene-benzothiadiazole-thiophene (5\%) moieties (Figure $1(\mathrm{c}))$ ) as guest. F8BT has been widely deployed as the emission layer in polymer LEDs, as a laser gain medium $^{[23,38-40]}$ and also as a resonance energy transfer host for red-light emission guest gain polymers, ${ }^{[41-42]}$ including Red F. ${ }^{[38,43-45]}$ The large fractional overlap between the emission spectrum of F8BT and the absorption spectrum of Red F leads to efficient Förster energy transfer, resulting in red emission from the blend (vide infra). Whilst Red $\mathrm{F}$ is not itself a LCCP, at $10 \mathrm{wt} . \%$ or less the F8BT host LCCP is still able to ensure polarized emission. 


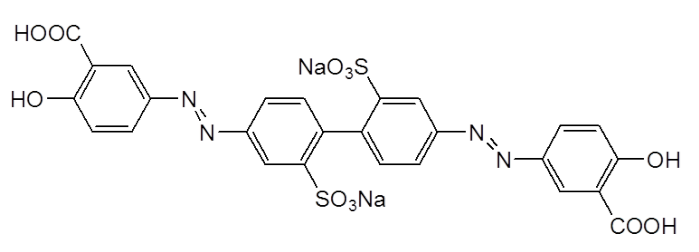

(a)

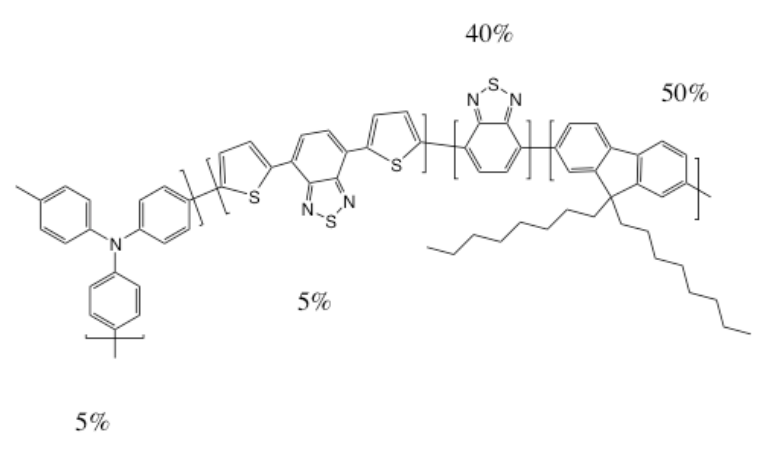

(c)

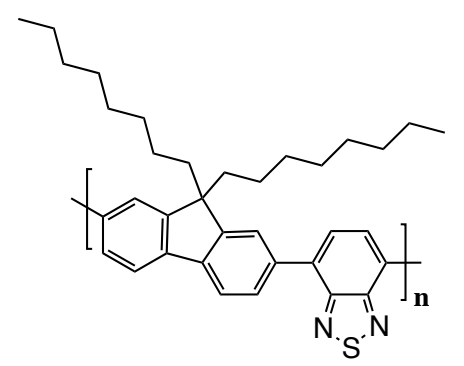

(b)

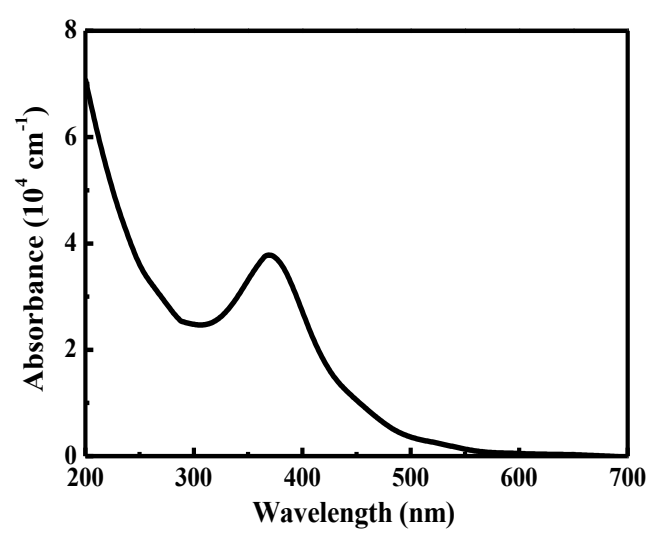

(d)

Figure 1: Chemical structures for (a) SD1, (b) F8BT and (c) Red-F. Also shown in (d) is the spectral profile of the absorbance for an SD1 thin film $(\sim 30 \mathrm{~nm})$ deposited on a quartz substrate.

\section{Results and discussion}

The process to generate patterned chain orientation structures for films of F8BT and F8BT/Red-F blend (deposited from a $90 \mathrm{wt} \% \mathrm{~F} 8 \mathrm{BT} / 10 \mathrm{wt} \%$ Red-F solution mixture) on SD1 coated Spectrosil substrates $\left(1.2 \times 1.2 \mathrm{~cm}^{2}\right)$ is outlined in Figure S1(a); see experimental section below for further details. Under polarized UV light SD1 tends to align with its long axis perpendicular to the incident polarization direction (order parameter $\mathrm{S} \sim 0.5$ ), ${ }^{[34]}$ providing an azimuthal anchoring energy comparable to that of rubbed polyimide films ( $\left.10^{-4} \mathrm{~J} \mathrm{~m}^{-2}\right) .{ }^{[46,47]}$ Thermal annealing then drives thermotropic homogeneous alignment of the LCCP atop the UV exposed SD1 regions and quenching freezes the resulting order into a nematic glass state. 


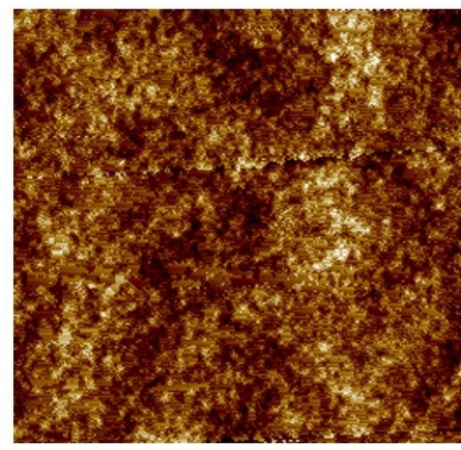

(a)

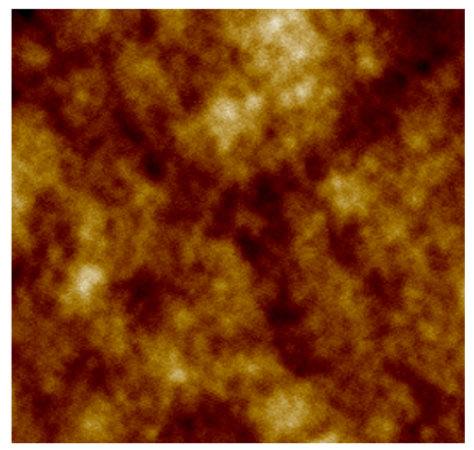

(c)

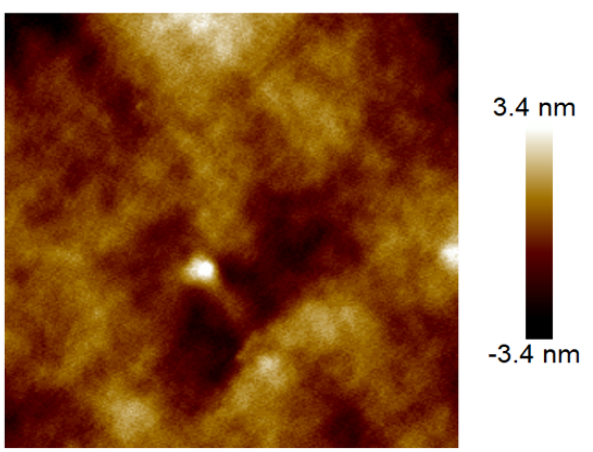

(b)

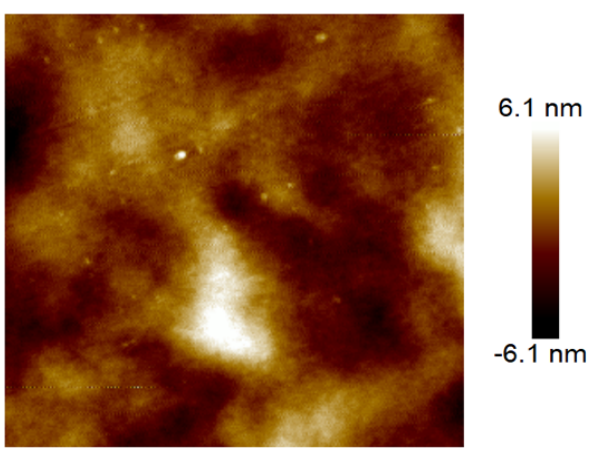

(d)

Figure 2: Atomic force microscope (AFM) film surface topography images $\left(3 \times 3 \mu \mathrm{m}^{2}\right.$ area) for: (a) Polarised UV photoexposed SD1 (30 nm thickness); (b) F8BT (250 nm) aligned on top of photoexposed SD1; (c) F8BT/Red-F blend (200 nm) aligned on top of photoexposed SD1; (d) F8BT double layer (150 nm each) separately aligned on top of photoexposed SD1 films.

Figure 2 shows atomic force microscope (AFM) images of surface topographies for (a) SD1, (b) aligned F8BT, and (c) aligned F8BT/Red-F blend films. The deduced root-mean-square (RMS) roughness values are $0.41 \pm 0.05 \mathrm{~nm}$ for $\mathrm{SD} 1,0.7 \pm 0.1 \mathrm{~nm}$ for F8BT and $0.7 \pm 0.1 \mathrm{~nm}$ for F8BT/Red-F, suggesting good uniformity despite the extensive processing required. Novel double layer structures were also fabricated involving a repeat of the process outlined in Figure S1(a), with spin-coating of a second SD1 layer on top of the first aligned conjugated polymer film after ozone treatment of the latter to increase adhesion. The resulting surface topography for such a F8BT double layer structure (Figure S1(b)) is shown in Figure 2d and reveals a still modest, albeit somewhat higher, RMS roughness $=1.3 \pm 0.2 \mathrm{~nm}$. 
The degree of molecular orientation in the processed LCCP films was examined using polarized absorption and photoluminescence (PL) spectroscopy. Spectra were recorded with incident light polarized both parallel $(/ /)$ and perpendicular $(\perp)$ to the polymer chain alignment direction and PL was collected without further polarization selection. Figures 3(a), (b) and (c) show typical spectral data, with evident anisotropy in all three cases; parallel-polarized incident light (solid line spectra) is more strongly absorbed and generates stronger PL. The absorption anisotropy, $A_{A b s}=\mathrm{Abs} / / \mathrm{Abs}_{\perp}$, measured at the low energy peak wavelength, $\lambda_{\max }$, ranges from $A \approx 3.5$ for the F8BT/Red-F blend (Figure $3(\mathrm{~b}), \lambda_{\max }=450 \mathrm{~nm}$ ), through $A_{A b s} \approx$ 7.25 for F8BT (Figure 3(a), $\lambda_{\max }=450 \mathrm{~nm}$ ), to $A_{A b s} \approx 11.4$ for the bilayer F8BT structure (Figure 3(c), $\lambda_{\max }=450 \mathrm{~nm}$ ). Previous results for F8BT aligned on rubbed polyimide ${ }^{[2,13,14]}$ fall within the same range, confirming the effectiveness of the SD1-based photoalignment process. In terms of $\mathrm{PL}$, we find anisotropy values $A_{P L}=\mathrm{PL}_{/ / /} \mathrm{PL}_{\perp} \approx 9$ (at $550 \mathrm{~nm}$ ) for $\mathrm{F} 8 \mathrm{BT}$, $A_{P L} \approx 5.1$ (at $635 \mathrm{~nm}$ ) for the F8BT/Red-F blend and $A_{P L} \approx 13.8$ (at $548 \mathrm{~nm}$ ) for the F8BT bilayer sample. A number of factors will influence the measured anisotropies. At a molecular level the angle that the optical dipole transition moment makes with the chain axis provides a fundamental limit on the observable anisotropy ${ }^{[1,13,14]}$. Other factors include the order parameter, $S$, achieved for chain axis orientation and the liquid crystalline domain structure adopted. ${ }^{[1,13]}$ For F8BT the dipole transition moment angle has been estimated to be $\approx 22^{\circ}$ which would suggest a maximum possible anisotropy, $A_{A b s}{ }^{\max }=12.25 .^{[13]}$ 

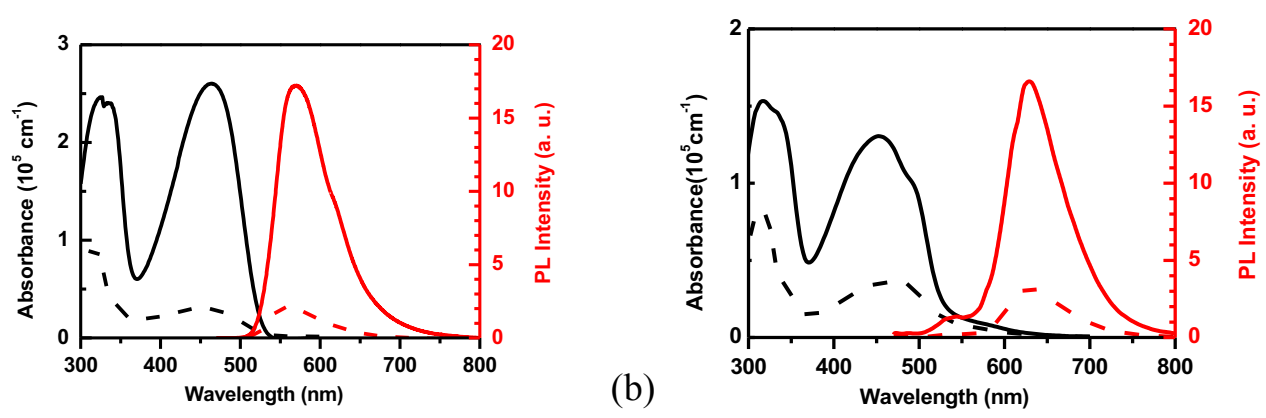

(c)
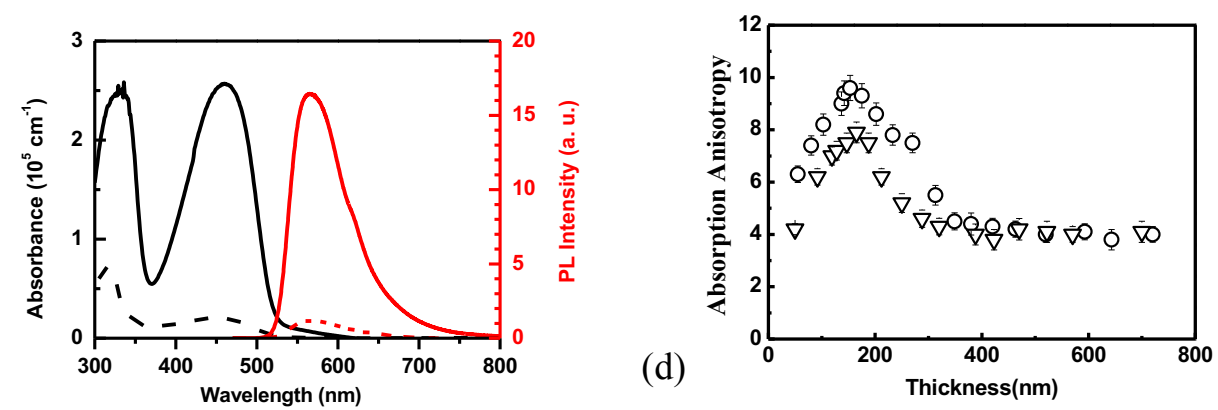

Figure 3: Polarized absorption (left ordinate) and PL (right ordinate) spectra for aligned (a) F8BT (255 nm thickness), (b) F8BT/Red-F blend (305 nm thickness) and (c) bi-layer F8BT films (140 nm thickness each). Solid line spectra were recorded with the incident light polarized parallel and dashed line spectra, perpendicular, to the polymer chain orientation direction. (d) Absorption anisotropy at $\lambda_{\max }$ as a function of thickness for F8BT (open circles data) and F8BT/Red-F blend (open down triangles data) films aligned on identically prepared SD1 photoalignment layer coated Spectrosil substrates. No account has been taken of the minor contribution (see Figure S1(d)) from the thin SD1 layer to these spectra.

The influence of LCCP film thickness on absorption anisotropy was carefully studied so as to better understand the scope for order parameter optimisation. The polymer chains within the nematic liquid crystalline phase behave in a cooperative manner with the alignment layer acting to direct their assembly, ideally into a specific, monodomain state. It is expected that the interplay between chain-chain and chain-alignment layer interactions will mediate the ordering; for thicker films there may be a tendency to order spontaneously, in ways no longer wholly directed by the alignment layer. Figure 3(d) shows the variation in $A_{A b s}$ for a set of F8BT (open circles data) and F8BT/Red-F blend (open down triangles data) films aligned on identically prepared SD1 photoalignment layer coated Spectrosil substrates and subject to the same straightforward annealing protocol (see experimental section for details). It is evident 
that alignment under these conditions is favoured for films with thicknesses in the range $\sim 100$ to $\sim 250 \mathrm{~nm}$; the peak anisotropy for F8BT is 9.7 at film thickness $150 \mathrm{~nm}$ whilst for the blend, $A_{A b s}$ reaches 8 at $155 \mathrm{~nm}$ thickness. It is also clear that the blend gives systematically lower anisotropies until film thicknesses $\geq 350 \mathrm{~nm}$ are used, at which point both film types have similar, albeit relatively low, anisotropies with $A_{A b s} \approx 4$. Further studies will be required to ascertain in detail the origins of this thickness variation but it reassuringly demonstrates that best orientation occurs for film thicknesses similar to those commonly used in a variety of device structures. ${ }^{[21,42,48,49]}$ The asymptotic limit for thicker films, were it to persist to $\mu \mathrm{m}$ scale, would be higher than the PL anisotropy achieved previously for $3 \mu \mathrm{m}$ thickness poly(9,9-dioctylfluorene) LCCP time-of-flight photocurrent samples aligned using a rather onerous annealing protocol on rubbed polyimide, where $A_{P L} \approx 2.4 \pm 0.2 .^{[24]}$

The highest anisotropies are seen for the SD1 (30 nm) / F8BT (140 nm) / SD1 (30 nm) / F8BT $\left(140 \mathrm{~nm}\right.$ ) double LCCP layer structures (Figure 3(c)). The slightly higher $A_{A b s}$ for the bilayer film $\approx 11.4$ versus $\approx 9.5$ for a corresponding $142 \mathrm{~nm}$ single layer F8BT film (Figure 3(d)) is not fully understood and was not anticipated given the high processing temperatures required and the use of SD1 on top of a conjugated polymer to which it might not be expected to adhere as well as it does to the Spectrosil substrate.

Deposition of the second SD1 layer is found to have no direct effect on the anisotropy of the first F8BT layer. It is possible, however, that the second thermal treatment helps to achieve further alignment. Even in the absence of a detailed explanation, this observation represents an encouraging result for the potential use of photoalignment to fabricate a variety of LCCP multilayer structures. In addition, this approach allows a higher anisotropy for thicker films (280 nm combined F8BT thickness in the bilayer) than available from an equivalent thickness single layer (c.f. Figure 3(d)), and also the opportunity to independently pattern the individual layers (vide infra). 
One of the specific advantages of using a photoalignment approach is the ability to generate spatial patterns in the LCCP orientation by masking the polarized UV light exposure of the SD1 film, thereby yielding a variety of optical structures. Figure 4(a) shows just such a pattern that was generated within a F8BT/Red-F film. The letters (standing for Nanjing University of Posts and Telecommunications Institute of Advanced Materials) correspond to aligned regions and are revealed by viewing the sample PL in a microscope equipped with crossed polarizers. The excitation light passing through the bottom polarizer (hatched red arrow) was polarized at $45^{\circ}$ to the polymer chain axis (solid white arrow). Further simple patterns are shown in Figure S2 for both F8BT/Red-F blend and F8BT LCCP films; in this case with orthogonal excitation light polarization so that the aligned regions appear dark. A patterning resolution test using a standard 1951 US Airforce Resolving Power Test Target as mask (see Figure S3) shows that $<4 \mu \mathrm{m}$ features are readily generated and Figure 4(b) shows a polarized PL image of a set of simple stripes with widths from 3 to $30 \mu \mathrm{m}$ (bottom to top, bright regions) and a fixed inter-stripe spacing of $10 \mu \mathrm{m}$. Chain orientation is perpendicular to the long axis of the stripe and the excitation light was polarized at $45^{\circ}$ to the chain axis. Good fidelity is obtained for the $3 \mu \mathrm{m}$ stripe, setting a further upper bound on the resolution limit. No smaller structures have been fabricated at this time but we note that an earlier study of SD1 showed that $\sim 100 \mathrm{~nm}$ orientation patterning can be achieved under specific conditions. ${ }^{[33]}$ 

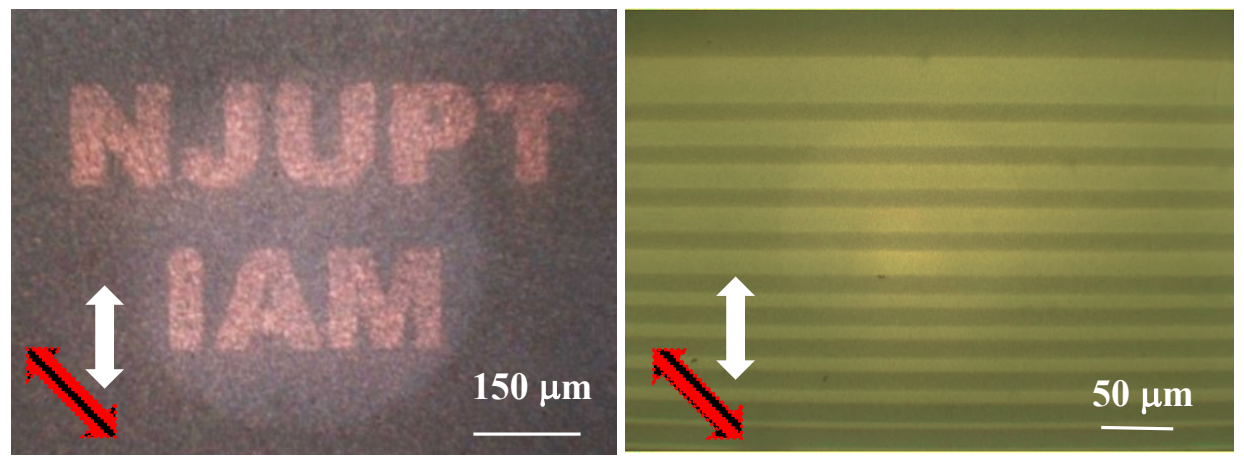

(a)

(b)
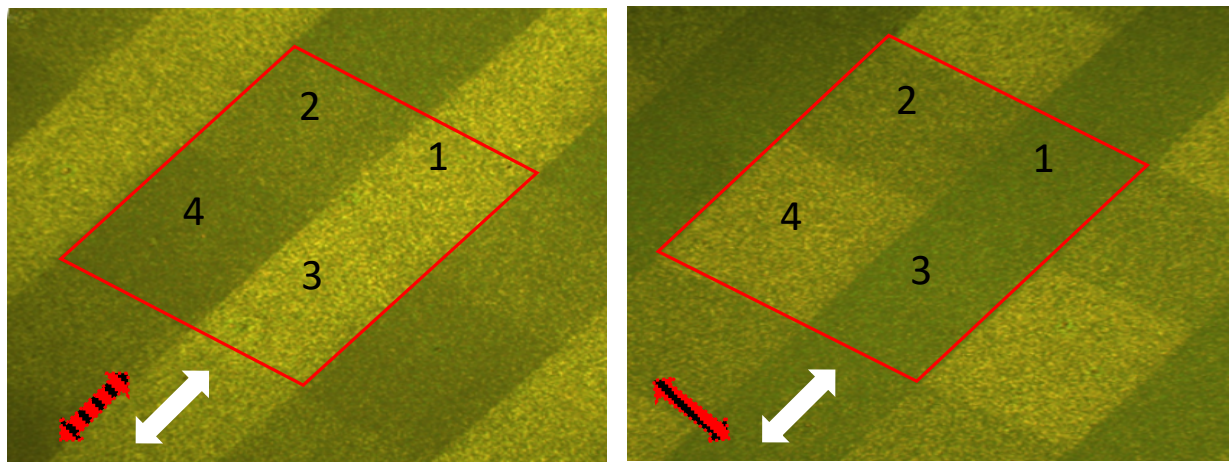

(c)

(d)

Figure 4: Chain-orientation-based optical structures generated for (a) an F8BT/Red-F blend film and (b) an F8BT film. The images show PL emission patterns observed with a polarizing microscope (c.f. bar for pattern scale) for back side illumination. Also shown ((c) and (d)) is an example of F8BT double layer patterning (see text for details) wherein one set of stripes is defined in a bottom F8BT film then a second set, orthogonal to the first, in a second F8BT film deposited on top. Double headed solid white arrow represents the molecular chain orientation and hatched red arrow represents the polarization direction of the excitation light.

F8BT double layer patterning was also demonstrated (Figure 4(c) and (d)). The schematic in Figure S1(c) shows the configuration of the chain alignment stripes and the nature of the pattern they delineate. The solid white arrows show the alignment direction for the upper F8BT layer and the red hatched arrows again show the bottom polarizer alignment. The PL excitation polarization in Figure 4 (c) is thus parallel to the diagonal top layer stripes (i.e. perpendicular to the bottom layer stripes) and in Figure 4 (d) perpendicular to the diagonal top layer stripes (i.e. parallel to the bottom layer stripes). This allows regulation of the emission intensity at different locations through selection of the relative orientation of the incident PL excitation, bottom and top film stripe directions and chain alignment relative to the stripes. 
Here, the polymer chains are aligned parallel to the stripes for both films. Four regions are delineated in Figure 4 (c) and Figure 4 (d): 1 and 3 overlap with a single aligned top stripe, 3 and 4 a single aligned bottom stripe, 2 is a region not aligned for either bottom or top film, 4 is not aligned for the top film and 1 not aligned for the bottom film. The widths of the dark non-aligned stripes and bright aligned stripes in Figure 4 (c) are, respectively, $265 \mu \mathrm{m}$ and $215 \mu \mathrm{m}$. Note also that the intensity levels are not matched between the two images; the automatic gain setting is higher for Figure 4 (d). Region 2 should yield a constant PL intensity.

Additional orientation patterns (c.f. scale bars for dimensions) are shown in Figure 5 (a) and (c), again imaged in PL emission using a microscope with crossed polarizers. The bottom polarizer alignment, indicated by the red hatched double headed arrow, defines the PL excitation polarization direction relative to the chain orientation direction (solid white arrow). The structure in Figure 5 (a) is akin to a multi-mode interference region with three output guides and was defined using a single SD1 exposure giving rise to a single orientation direction throughout. Figure S4 shows a sequence of images for an equivalent structure with different relative alignment between the excitation polarization and chain orientation, resulting in states with different characteristic visibilities including both dark and invisible (against the non-oriented background). In relation to the PL anisotropy (Figure 5(b)), we found $A_{P L} \approx 7-8$ in the oriented region and $A_{P L} \approx 1.1$ in the non-oriented region for a film thickness of $260 \mathrm{~nm}$. The latter small anisotropy is believed to be an experimental artefact from the measurement geometry. 


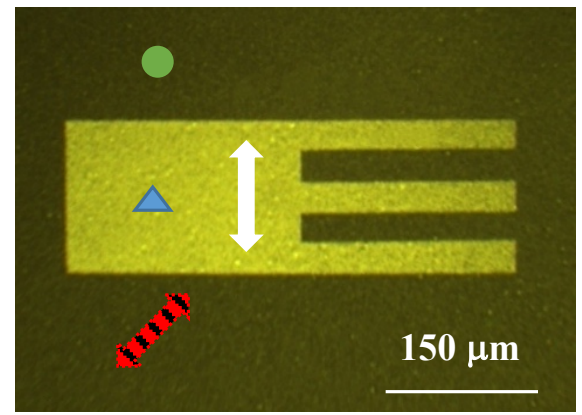

(a)

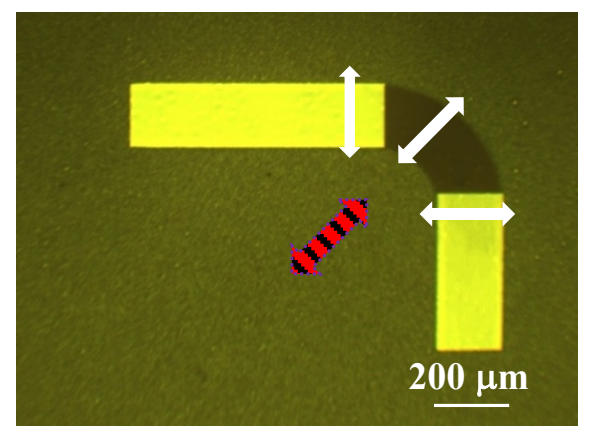

(c)

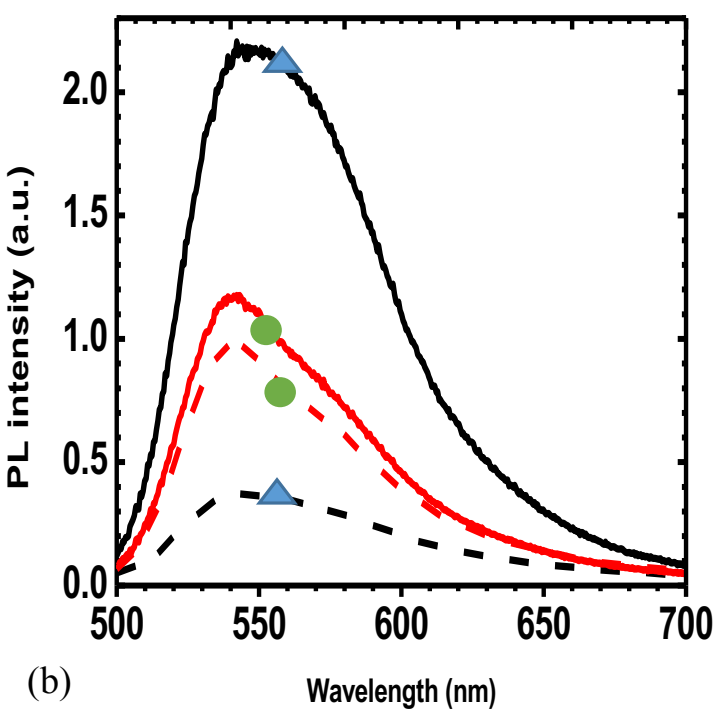

Figure 5. Further chain-orientation-based optical structures generated in F8BT films. The image in (a) is for a specific bright state of a monolithic structure. Polarised PL spectra are shown in (b) for both the oriented region (identified by a blue filled up-triangle) and a nonoriented region (identified by a green filled circle). In (c) a three-part structure is shown comprising two orthogonal stripes linked by a curvilinear section. Here the selection of chain orientation and excitation polarization directions results in a bright state for both of the orthogonal stripes and a dark state for the linker region.

The structure in Figure 5 (c) was prepared by subjecting the SD1 photoalignment layer to three separate (masked) exposures to generate the three sections, using polarization angles $0^{\circ}$ (first linear stripe section), $45^{\circ}$ (curvilinear linker section) and $90^{\circ}$ (second linear stripe section). The image shown is for PL excitation polarised at $45^{\circ}$ (red hatched double headed arrow), parallel to the chain alignment direction within the curvilinear region (solid white arrow). A dark state results as this polarisation is orthogonal to the top polarizer. Conversely, the linear stripe regions are both in a bright state as the PL excitation polarisation is at $45^{\circ}$ to their chain alignment direction and to the top polarizer. Figure S5 shows angle dependent 
measurements for an equivalent structure written again with three SD1 exposures, this time using polarization angles $0^{\circ}, 22.5^{\circ}$ and $45^{\circ}$.

The ability to write patterns offers the possibility to fabricate photonic structures such as waveguides and other light propagation control structures (e.g. gratings and diffractive elements). ${ }^{[44,45]}$ Here, the observed anisotropy in absorption due to chain orientation will be mirrored, in accord with the Kramers-Kronig relations, in a corresponding anisotropy in film refractive index (n). This has previously been used to control the emission from microcavity structures, leading to a splitting in resonances for parallel and perpendicular polarized light that produces exceptional polarization ratios at the resonance wavelengths. ${ }^{[20]}$

The refractive index of spin-coated F8BT at $577 \mathrm{~nm}$, the peak optical gain wavelength, has been reported to be $1.69 .{ }^{[23]}$ For films aligned on rubbed polyimide the index along the polymer chain direction increases to 2.1 , while the perpendicular index reduces to $\approx 1.59$. ${ }^{[14,23]}$ The resulting index difference is significantly higher than for the conformation-changepatterning we have previously reported, which was already sufficient to generate useful optical confinement. ${ }^{[50,51]}$ The added complication here is the anisotropy, which means that different polarisations of light experience different indices even when propagating in the same direction; this makes design of structures more involved and rules out certain applications. However, in common with the conformation-change approach, patterning via chain alignment is film thickness conserving, supporting an attractive planar architecture.

As a demonstration of the sorts of structures one might fabricate, Figure 4(b) showed a set of simple stripes with width down to $3 \mu \mathrm{m}$, whilst Figure 5 shows more complex structures fabricated with both a single SD1 exposure step for a unidirectional linear structure (Figure 5(a)) and a three-fold SD1 exposure sequence to accommodate a stripe that undergoes a $90^{\circ}$ change in direction with a curvilinear connecting region (Figure 5(c)). Also shown in 
Figure 5(b) are the polarized excitation PL spectra recorded at specific locations both within and without the aligned region; these are wholly consistent with the data shown in Figure 3(a).

Finally, it should be emphasized that no studies have yet been undertaken to explore light propagation in such structures and that several requirements will need to be met before that can be done. Most critical will be to improve the optical quality of the films through removal of impurities (including dust) from the starting materials and through management of the processing to avoid film heterogeneities. The latter can arise through partial crystallization during thermal processing, voids induced by trapped solvent, and other variations in microstructure, especially for blend films. Grating or prism couplers will also generally be needed as butt-coupling is difficult for thin film formats due to mode-matching challenges and especially so for soft materials that do not cleave readily to form a well-defined facet.

\section{Summary and Conclusions}

SD1 has been shown to be an effective, high-temperature stable $\left(\geq 265^{\circ} \mathrm{C}\right)$, photoalignment material for nematic LCCP orientation. It performs as well as previously reported rubbed polyimide films, with both oriented F8BT and F8BT/Red F blend samples showing strong optical anisotropies. The latter range from $A_{A b s}=3.5$ for the blend through to11.4 for an F8BT bilayer, with optimal LCCP film thickness found to lie within the device relevant 125 to 200 nm range.

Spatial patterning, with linear and curvilinear features and linewidths down to $\sim 3 \mu \mathrm{m}$, has also been straightforwardly demonstrated, including independent sequential orientation of films on top of each other. This is not feasible with a rubbed polyimide alignment approach. Furthermore, multiple PL optical states, defined by the relative alignment between chain orientation direction and PL excitation and detection polarization are readily created with 
visibilities that range from dark to bright (relative to the unaligned background), including invisible when the pattern and background show matched PL intensities.

Photoalignment also provides a route to enhancing the coupling strength in ultrastrongly coupled microcavities where the orientation of the polymer chains increases the vector dot product $(\boldsymbol{\mu} . \boldsymbol{E})$ between the optical transition dipole moment $(\boldsymbol{\mu})$ and a suitably polarised optical electric field vector $(\boldsymbol{E}) \cdot{ }^{[52]}$ For F8BT this leads to a coupling ratio $g=$ Rabi splitting energy $\left(E_{\text {Rabi }}\right) /$ optical transition energy $\left(E_{e x}\right)=1.25 \mathrm{eV} / 2.71 \mathrm{eV}=46 \%$ that is substantially larger than the $\approx 10-20 \%$ threshold for ultrastrong coupling. Similar behaviour is seen for poly(9,9-dioctylfluorene) (PFO) where $g=1.47 \mathrm{eV} / 3.23 \mathrm{eV}=46 \%$, whilst for poly(9,9dihexylfluorene-co-dithiophene) (F6T2) $g=1.80 \mathrm{eV} / 2.79 \mathrm{eV}=65 \%$, the highest reported value to date for an organic cavity. In the latter case the Rabi splitting of $1.8 \mathrm{eV}$ also puts the inter-polariton transition within the visible spectrum for the first time.

The availability of such a versatile photoalignment material for LCCPs offers many opportunities to tune the electronic and optical properties of this class of polymer semiconductor for different device applications and our future work will seek to address a number of these opportunities.

\section{Experimental section}

The SD1 photoalignment material used in this study was purchased from the Nanjing Murun Advanced Material Company Ltd and used as supplied. F8BT $\left(\mathrm{M}_{\mathrm{w}}=55,000\right)$ was purchased from Hanfeng Ltd and Red-F was supplied by the Sumitomo Chemical Company Ltd; again, both were used as supplied. The chemical structures of SD1, F8BT and Red-F are shown in supporting information Figure 1. 
Chain oriented F8BT and F8BT/Red-F blend films were fabricated using SD1 as the photoalignment layer. The four-step procedure (Figure S1a) comprised:

(i) Spin-coating $30 \mathrm{~nm}$ thickness SD1 films from $5 \mathrm{mg} / \mathrm{ml}$ dimethylformamide solution onto pre-cleaned polished Spectrosil substrates and annealing at $100{ }^{\circ} \mathrm{C}$ for $10 \mathrm{~min}$.

(ii) Masked exposure of the SD1 films to $\geq 5 \mathrm{~J} / \mathrm{cm}^{2}$ linearly polarised (Glan-Taylor prism) ultraviolet (405 nm) light (for example using $10 \mathrm{~mW} / \mathrm{cm}^{2}$ for $10 \mathrm{~min}$ ).

(iii) Spin-coating 50 - $720 \mathrm{~nm}$ thickness LCCP and LCCP blend films on top. For F8BT spincoating was from $20-30 \mathrm{mg} / \mathrm{ml}$ toluene solution and for F8BT/Red-F blend films 20 - 30 $\mathrm{mg} / \mathrm{ml}$ toluene solutions of each were mixed in a ratio 9:1 F8BT:Red-F prior to spin-coating. (iv) Annealing in a Linkam LTS420E-PB4 hot stage at $265^{\circ} \mathrm{C}$ for 2 min under nitrogen, followed by slow cooling at $1{ }^{\circ} \mathrm{C} / \mathrm{min}$ to $235^{\circ} \mathrm{C}$ and then rapid quenching to room temperature.

For bilayer samples the process was repeated for the second LCCP film (see Figure S1b for schematic), with the first oriented LCCP film exposed to ozone (generated using a 120W, 254 nm UV lamp) for 30 min before depositing SD1 on top.

Film thicknesses were measured with a Bruker Dektak XT stylus profilometer and film surface topography with a Bruker Dimension Icon atomic force microscope (AFM) equipped with Scanasyst-Air peak-force tapping mode tips. Film absorption and PL spectra were measured, respectively, using Perkin-Elmer Lambda 35 UV/Vis spectrophotometer and LS 55 Fluorescence spectrometer with excitation wavelength set to $450 \mathrm{~nm}$.

\section{Supporting Information}

Supporting Information is available from the Wiley Online Library or from RX.

\section{Acknowledgments}

We thank Takeshi Yamada and the Sumitomo Chemical Company for providing the Red-F polymer. We further thank Mariano Campoy-Quiles for assistance with refractive index 
measurements. R.X. acknowledges funding from the National Natural Science Foundation of China (Grants 61874058, 51861145301 and 61376023), a Chinese 973 project (grant 2015CB932203), and the Priority Academic Program Development Fund of Jiangsu Higher Education Institutions (PAPD-YX030003). DDCB acknowledges the University of Oxford and the Jiangsu Province Double Creation Team Award for funding support. YS thanks the Hong Kong Jockey Club for a DPhil Graduate Scholarship.

\section{References}

[1] M. Grell, D. D. C. Bradley, M. Inbasekaran, E. P. Woo, Adv. Mater. 1997, 9, 798.

[2] M. Grell, M. Redecker, K. Whitehead, D. D. C. Bradley, M. Inbasekaran, E. P. Woo, Liq. Cryst. 1999, 26, 1403.

[3] K. Sakamoto, T. Yasuda, K. Miki, M. Chikamatsu, R. Azumi, J. Appl. Phys. 2011, 109, 013702.

[4] J. W. Bae, K. Song, Org. Electron. 2016, 30, 143.

[5] M. Grell, D. D. C. Bradley, X. Long, T. Chamberlain, M. Inbasekaran, E. P. Woo, M. Soliman, Acta Polym. 1998, 49, 439.

[6] M. Grell, D. D. C. Bradley, G. Ungar, J. Hill, K. Whitehead, Macromolecules. 1999, 32,5810 .

[7] M. Grell, W. Knoll, D. Lupo, A. Meisel, T. Miteva, D. Neher, H.-G. Nothofer, U. Scherf, A. Yasuda, Adv. Mater. 1999, 11, 671.

[8] M. Gather, M. Heeney, W. Zhang, K. Whitehead, D. D. C. Bradley, I. McCulloch, A. J. Campbell, Chem. Commun. 2008, 9, 1079.

[9] S.-H. Yang, C.-S. Hsu, J. Poly, Sci. Part A: Poly. Chem. 2009, 47, 2713.

[10] M. Grell, D. D. C. Bradley, Adv. Mater. 1999, 11, 895.

[11] Y. Kim, N. Minami, S. Kazaoui, Appl. Phys. Lett. 2005, 86, 073103.

[12] K. S. Whitehead, M. Grell, D. D. C. Bradley, M. Jandke, P. Strohriegl, Appl. Phys.

Lett. 2000, 76, 2946. 
[13] H. Liem, P. Etchegoin, K. S. Whitehead, D. D. C. Bradley, Adv. Funct. Mater. 2003, 13,66 .

[14] M. Campoy-Quiles, P. G. Etchegoin, D. D. C. Bradley, Phys. Rev. B. 2005, 72, 045209 .

[15] S. F. Chung, T. C. Wen, W. Y. Chou, T.-F. Guo, Jpn. J. Appl. Phys. 2006, 45, L60.

[16] M. C. Gather, D. D. C. Bradley, Adv. Funct. Mater. 2007, 17, 479.

[17] Z. Zheng, K. H. Yim, M. S. Saifullah, M. E. Welland, R. H. Friend, J. S. Kim, W. T. Huck, Nano Letters. 2007, 7, 987.

[18] K. Chung, Y. Yu, M. S. Kwon, J. Swets, J. Kim, J. H. Youk, MRS Commun. 2015, 5, 169.

[19] a) K. S. Whitehead, M. Grell, D. D. C. Bradley, M. Jandke, P. Strohriegl, SPIE

Proceedings. 2000, 3939, 172. b) M. P. Aldred, A. E. A. Contoret, S. R. Farrar, S. M. Kelly,

D. Mathieson, M. O'Neill, W. C. Tsoi, P. Vlachos, Adv. Mater., 2005, 17, 1368-1372.

[20] T. Virgili, D. G. Lidzey, M. Grell, S. Walker, A. Asimakis, D. D. C. Bradley, Chem. Phys. Lett. 2001, 341, 219.

[21] T. Virgili, D. G. Lidzey, M. Grell, D. D. C. Bradley, S. Stagira, M. Zavelani-Rossi, S. De Silvestri, Appl. Phys. Lett. 2002, 80, 4088.

[22] G. Heliotis, R. Xia, K. S. Whitehead, G. A. Turnbull, I. D. W. Samuel, D. D. C. Bradley, Synth. Met. 2003, 139, 727.

[23] R. Xia, M. Campoy-Quiles, G. Heliotis, P. Stavrinou, K. S. Whitehead, D. D. C. Bradley, Synth. Met. 2005, 155, 274.

[24] M. Redecker, D. D. C. Bradley, M. Inbasekaran, E. P. Woo, Appl. Phys. Lett. 1999, 74, 1400.

[25] H. Sirringhaus, R. J. Wilson, R. H. Friend, M. Inbasekaran, E. P. Woo, M. Grell, D. D. C. Bradley, Appl. Phys. Lett. 2000, 77, 406.

[26] T. Endo, T. Nagase, T. Kobayashi, H. Naito, Jpn. J. Appl. Phys. 2013, 52, 121601. 
[27] H. Y. Noh, C. S. Park, J. S. Park, S. W. Kang, H. R. Kim, Jpn. J. Appl. Phys. 2012, 51, 06FJ03.

[28] K. Fukuhara, S. Nagano, M. Hara, T. Seki, Nature Communications, 2014, 5, 3320.

[29] D. Sainova, A. Zen, H.-G. Nothofer, U. Asawapirom, U. Scherf, R. Hagen, T.

Bieringer, S. Kostromine, D. Neher, Adv. Funct. Mater. 2002, 12, 49.

[30] a) T. Fujiwara, J. Locklin, Z. Bao, Appl. Phys. Lett. 2007, 90, 232108. b) A. E. A.

Contoret, S. R. Farrar, P. O. Jackson, S. Khan, L. May, M. O'Neill, J. E. Nicholls, S. M. Kelly, G. J. Richards, Adv. Mater. 2000, 12, 971.

[31] T. Seki, S. Nagano, M. Hara, Polymer. 2013, 54, 6053.

[32] O. Yaroshchuk, Y. Reznikov, J. Mater. Chem. 2012, 22, 286.

[33] E. A. Shteyner, A. K. Srivastava, V. G. Chigrinov, H.-S. Kwok, A. D. Afanasyev, Soft Matter. 2013, 9, 5160.

[34] V. Chigrinov, H. S. Kwok, H. Takada, H. Takatsu, Liq. Cryst. Today. 2005, 14, 1.

[35] L. L. Ma, M. J. Tang, W. Hu, Z. Q. Cui, S. J. Ge, P. Chen, L. J. Chen, H. Qian, L. F. Chi, Y. Q. Lu, Adv. Mater. 2017, 29, 6671.

[36] L. L. Ma, S. S. Li, W. S. Li, W. Ji, B. Luo, Z. G. Zheng, Z. P. Cai, V. Chigrinov, Y. Q. Lu, W. Hu, L. J. Chen, Adv. Opt. Mater. 2015, 3, 1691.

[37] W. Hu, A. Kumar Srivastava, X. W. Lin, X. Liang, Z. J. Wu, J. T. Sun, G. Zhu, V. Chigrinov, Y. Q. Lu, Appl. Phys. Lett. 2012, 100, 111116.

[38] R. Xia, G. Heliotis, Y. B. Hou, D. D. C. Bradley, Org. Electron. 2003, 4, 165.

[39] Q. Zhang, Q. Wei, X. Guo, G. Hai, H. Sun, J. Li, R. Xia, Y. Qian, S. Casado, J. R. Castro-Smirnov, J. Cabanillas-Gonzalez, Adv. Sci. 2018, 5, 1801455.

[40] Q. Zhang, J. Liu, Q. Wei, X. Guo, Y. Xu, R. Xia, L. Xie, Y. Qian, C. Sun, L. Lüer, J. Cabanillas-Gonzalez, D. D. C. Bradley, W. Huang, Adv. Funct. Mater. 2018, 28, 1705824. 
[41] Y. Xu, G. Hai, H. Xu, H. Zhang, Z. Zuo, Q. Zhang, R. Xia, C. Sun, J. Castro-Smirnov, A. Sousaraei, S. Casado, M. R. Osorio, D. Granados, I. Rodriguez, J. Cabanillas-Gonzalez, Adv. Opt. Mater. 2018, 6, 1800263.

[42] R. Xia, P. N. Stavrinou, D. D. C. Bradley, Y. Kim, J. Appl. Phys. 2012, 111, 123107.

[43] G. Heliotis, R. Xia, D. D. C. Bradley, G. A. Turnbull, I. D.W. Samuel, P. Andrew, W. L. Barnes, J. Appl. Phys. 2004, 96, 6959.

[44] M. Campoy-Quiles, G. Heliotis, R. Xia, M. Ariu, M. Pintani, P. Etchegoin, D. D. C. Bradley, Adv. Funct. Mater. 2005, 15, 925.

[45] H. Yoon, S.A. Maier, D. D. C. Bradley, P. N. Stavrinou, Opt. Mater. Express. 2011, 1, 1127.

[46] M. Brinkmann, L. Hartmann, L. Biniek, K. Tremel, N. Kayunkid, Macromol. Rapid Commun. 2014, 35, 9 .

[47] M. A. Fourati, C. Pellerin, C. G. Bazuin, R. E. Prud'homme, Polymer, 2013, 54, 730.

[48] C. I. Wilkinson, D. G. Lidzey, L. C. Palilis, R. B. Fletcher, S. J. Martin, X. Wang, D.

D. C. Bradley, Appl. Phys. Lett. 2001, 79, 171.

[49] R. Xia, C. Cheung, A. Ruseckas, D. Amarasinghe, I. D. W. Samuel, D. D. C. Bradley, Adv. Mater. 2007, 19, 4054.

[50] G. Ryu, P. N. Stavrinou, D. D. C. Bradley, Adv. Funct. Mater. 2009, 19, 3237.

[51] A. Perevedentsev, Y. Sonnefraud, S. Sharma, A.E.G. Cass, S. A. Maier, J. S. Kim, P. N. Stavrinou, D. D. C. Bradley, Nat. Commun. 2015, 6, 5977.

[52] F. Le Roux, R. A. Taylor, D. D. C. Bradley, arXiv:1909.06158v1 [physics.app-ph] 13 Sep 2019. 
The table of contents entry:

Spatial patterning, with linear and curvilinear features and linewidths down to $\sim 3 \mu \mathrm{m}$, has been achieved with both chain oriented F8BT and a F8BT/Red F blend using SD1 as a photoalignment material. The chain-oriented areas show strong optical anisotropies, allowing the fabrication of both single- and multi-layer liquid crystalline conjugated polymer optical structures.

Keywords: Liquid crystalline conjugated polymer, F8BT and Red-F, SD1 photoalignment, Chain orientation optical structures, Refractive index modulation

Haoran Zhang, ${ }^{\#}$ Lingling Ma, Qian Zhang, Yuping Shi, Yueting Fang, Ruidong Xia, * Wei $H u$, Donal D. C. Bradley*

Azobenzene sulphonic dye photoalignment as a means to fabricate liquid crystalline conjugated polymer chain-orientation-based optical structures
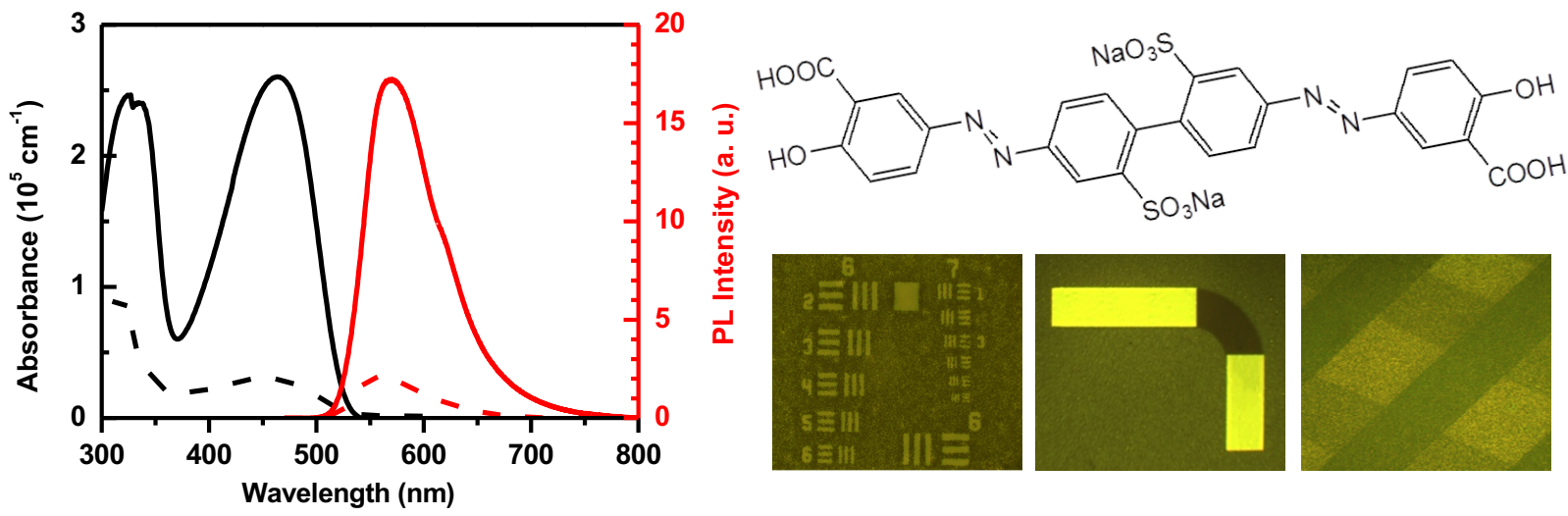